\title{
BONNESEN-STYLE INEQUALITIES FOR MINKOWSKI RELATIVE GEOMETRY
}

\author{
J. R. SANGWINE-YAGER
}

\begin{abstract}
Two Bonnesen-style inequalities are obtained for the relative inradius of one convex body with respect to another in $n$-dimensional space. Both reduce to the known planar inequality; one sharpens the relative isoperimetric inequality, the other states that a quadratic polynomial is negative at the inradius. Circumradius inequalities follow.
\end{abstract}

I. Introduction. By a convex body in Euclidean $n$-space, $E^{n}$, we will mean a compact convex set with nonempty interior. The unit ball centered at the origin will be denoted $B$, and $E$ will represent a fixed convex body. The Minkowski sum of two convex sets $K$ and $L$ is denoted $K+L$, and the multiple of $K$ by the scalar $t$ is $t K$. Steiner's formula states that

$$
V(K+t L)=\sum_{i=0}^{n}\left(\begin{array}{c}
n \\
i
\end{array}\right) t^{i} V(K \underbrace{\ldots, \ldots,}_{n-i} K, L \underbrace{, \ldots, L}_{i} L)
$$

where $V(\cdot)$ is the $n$-dimensional volume and the terms

$$
V(K \underbrace{\ldots \ldots, K}_{n-i} K, L \underbrace{, \ldots, L}_{i} L)
$$

called mixed volumes, are defined by this formula. For a discussion of mixed volumes see [11, p. 84 or 17, p. 209]. If $L=B$, then the mixed volume with $i$ copies of $B, i=0, \ldots, n$, is the ith quermassintegral, $W_{i}(K)$, of $K$. The zeroth quermassintegral is volume, $n$ times the first is surface area, the $(n-1)$ st is proportional to the mean width of $K$ where $W_{n-1}(B)=V(B)$, and the $n$th is constant and equal to $V(B)$.

The setting for this paper is Minkowski relative geometry. Here we use the Euclidean metric, but the functions of convex sets in which we will be interested, for example quermassintegrals and inradius, are evaluated relative to the fixed body $E$ rather than the unit ball $B$. The ith relative quermassintegral of a convex set $K, i=0, \ldots, n$, is defined by

$$
W_{i}(K ; E)=V(K \underbrace{\ldots \ldots}_{n-i} K, E \underbrace{\ldots, \ldots}_{i} E) .
$$

In most cases we will use $V(K)$ and $V(E)$ rather than the notation above for the zeroth and $n$th relative quermassintegrals. The relative inradius, $r(K ; E)$, and

Received by the editors April 8, 1987.

1980 Mathematics Subject Classification (1985 Revision). Primary 52A40.

Key words and phrases. Circumradius, convex body, inner parallel body, inradius, quermassintegral. 
circumradius, $R(K ; E)$, of $K$ with respect to $E$ are defined by

$$
\begin{aligned}
r(K ; E) & =\sup \{r: \text { some translate of } r E \subseteq K\}, \\
R(K ; E) & =\inf \{R: \text { some translate of } K \subseteq R E\} .
\end{aligned}
$$

Notice that if $K$ is a convex body

$$
r(K ; E)=1 / R(E ; K) .
$$

A convex set $K$ is said to be a homothet of $L$ if $K$ is a translate of a scalar multiple of $L$, that is $K=x+t L$, for some $x$ in $E^{n}$ and some scalar $t$. A convex body is said to be normal if the origin is an interior point. The kernel of $K$ with respect to a normal convex body $E$ will be denoted $K_{0}$ where

$$
K_{0}=\{x: x+r(K ; E) E \subseteq K\} .
$$

Note that the dimension of the kernel is less than $n$. If $E=B$, the kernel is the locus of the centers of incircles of $K$.

II. Known Bonnesen and Favard inequalities. An inequality of Bonnesen in the plane states

$$
0 \geq V(K)-2 t W_{1}(K ; E)+t^{2} V(E), \quad r(K ; E) \leq t \leq R(K ; E) .
$$

Equality holds at $t=r(K ; E)$ if and only if $K=K_{0}+r(K ; E) E$, and at $t=R(K ; E)$ if and only if $K=E$ or $K=\{x\}$. Bonnesen [7] proved this result for $E=B$; the proof for the relative case was established by Blaschke [2, pp. 33-36] and may also be found in [14]. This result is of special interest because it implies

$$
W_{1}^{2}(K ; E)-V(K) V(E) \geq\left(\frac{V(E)}{2}(R(K ; E)-r(K ; E))\right)^{2}
$$

which strengthens the relative isoperimetric inequality in the plane.

Several authors have investigated the extension of (2) to higher dimensions. The isoperimetric inequality was strengthened by Hadwiger [15], for $n=3$, and Dinghas [9], for all $n$, with the following inradius inequality

$$
\left(\frac{W_{1}(K)}{V(B)}\right)^{n /(n-1)}-\frac{V(K)}{V(B)} \geq\left(\left(\frac{W_{1}(K)}{V(B)}\right)^{1 /(n-1)}-r(K ; B)\right)^{n}
$$

where equality holds for $n=2$ if and only if $K=K_{0}+r(K ; B) B$, and for $n>2$ if and only if $K$ is a ball. If $n=2$, this inequality is (2) for $E=B$ and $t=r(K ; B)$. Dinghas' proof uses the method of inner parallel bodies and may be extended to relative inner parallel bodies; the extension of (3) to relative geometry will be given below as the first corollary of Theorem (19). Another extension of Bonnesen's inequality was conjectured by Wills [22], and proved by Bokowski [3] and Diskant [10]. It states

$$
0 \geq V(K)-n r(K ; B) W_{1}(K)+(n-1) r^{n}(K ; B) V(B)
$$

where for $n>2$ equality holds if and only if $K$ is a ball. Notice that if $n=2$ this inequality is also (2) for $E=B$ and $t=r(K ; B)$. The inequality was later sharpened by Osserman [18]. A further improvement, and the establishment of the inequality for relative geometry, will be presented as the first corollary to Theorem 
(23) below. For a discussion of Bonnesen inequalities, including nonconvex sets, see [18].

For $n$ larger than 2, (3) and (4) vary from the Bonnesen inradius inequality in the plane in two significant ways: equality does not hold for bodies of the form $K_{0}+$ $r(K ; B) B$, and the inequalities are not valid on the closed interval $[r, R]$. The first difference was the motivation for Theorem (23) and, for $n=3$, this result appears in an earlier paper by the author [19]. Because both inradius inequalities proved in this paper will be established for relative geometry, we may use (1) to obtain circumradius inequalities; these inequalities are stated as the second corollary to each theorem. There remain no known inequalities valid on the closed interval $[r, R]$; in the conclusion the author will state a conjecture due to Teissier [21] which involves this interval and is the third type of inequality which reduces to (2) if $n=2$.

Let $K$ be a planar convex body; for simplicity let $A=V(K)$, the area of $K$, and $L=2 W_{1}(K)$, the perimeter of $K$. An inequality of Favard [12, p. 362] states

$$
0 \leq A-2 R(K ; B) L+3 R^{2}(K ; B) \pi
$$

where equality holds if and only if $K$ is a ball. Notice that the direction of inequality sign is reversed from (2). Combining Favard's inequality, (2) for $E=B$ and $t=r(K ; B)$, and the simple inequality $2 A \geq r(K ; B) L$ we have

$$
\begin{gathered}
\left(L-\sqrt{L^{2}-4 \pi A}\right) / 2 \pi \leq r(K ; B) \leq 2 A / L, \\
\left(L+\sqrt{L^{2}-3 \pi A}\right) / 3 \pi \leq R(K ; B) \leq\left(L+\sqrt{L^{2}-4 \pi A}\right) / 2 \pi .
\end{gathered}
$$

These bounds for $r(K ; B)$ and $R(K ; B)$ can be shown to be best. In addition to establishing extensions of Bonnesen's inequality, bounds for the relative inradius and circumradius similar to those above will be discussed in the conclusion. The extension of Favard's inequality for $n$ greater than 2 was obtained by Bokowski and Heil [4]. If we let $R=R(K ; B), i, j$ and $k$ be integers such that $0 \leq i<j<k \leq n$, and $c_{i j k}=(i+1)(k-j)$, then their inequality states

$$
0 \leq c_{i j k} R^{i} W_{i}(K)+c_{j k i} R^{j} W_{j}(K)+c_{k i j} R^{k} W_{k}(K)
$$

where among convex bodies equality only holds for balls. Bokowski and Heil show that the coefficients used in the inequality are best. This inequality is not valid in relative geometry.

III. Inner parallel bodies and tangent bodies. The supporting function, $h(K, u)$, of a convex body $K$ in the direction of the unit vector $u$ is the signed distance from the origin to the supporting hyperplane of $K$ with outer normal $u$. A boundary point of $K$ is said to be extreme if it is not contained in the interior of any line segment contained in $K$. The Krein-Milman theorem states that every convex body is the convex hull of its extreme points, see [11, p. 24]. A supporting halfspace of a normal convex body $K$ is extreme if it is the dual of an extreme boundary point of the dual of $K$; the unit outer normal of the supporting halfspace is called an extreme direction of $K$. In general, $u$ is an extreme direction of a convex body if it is an extreme direction of a normal translate of the body. An alternate definition may be found in [11, p. 27]. The set of extreme unit vectors of $K$ will 
be denoted by $U$. The result which we will use is the dual of the Krein-Milman theorem which states

$$
K=\bigcap_{u \in U}\{x:\langle x, u\rangle \leq h(K, u)\}
$$

where $\langle\cdot, \cdot\rangle$ is the usual inner product.

A convex body is called regular by Aleksandrov [1,1/34-35] if it has at each point of its surface well-defined principal radii of curvature which are nowhere zero and continuous functions of the normal. The theorem below follows from a generalized version of the Brunn-Minkowski Theorem proved by Aleksandrov [1, 4/36]. To obtain the theorem we also use the fact that the $(n-i)$ th root of the $i$ th relative quermassintegral is monotone and homogeneous.

(8) THEOREM (AlEKSANDROV). Let $L, K_{1}, K_{2}$ and $E$ be convex bodies, let $i=0, \ldots, n-2$ and assume, for scalars $s$ and $t$, that $L \supseteq s K_{1}+t K_{2}$. Then

$$
\sqrt[n-i]{W_{i}(L ; E)} \geq s \sqrt[n-i]{W_{i}\left(K_{1} ; E\right)}+t \sqrt[n-i]{W_{i}\left(K_{2} ; E\right)}
$$

If $E$ is regular, equality holds if and only if $K_{1}$ and $K_{2}$ are homothetic and $L=$ $s K_{1}+t K_{2}$.

On several occasions we will use the following generalization of Steiner's formula. If $K, L$, and $E$ are convex sets, $i=0, \ldots, n$, and $s$ and $t$ are scalars, then

$$
\begin{aligned}
& W_{i}(s K+t L ; E) \\
& =\sum_{j=0}^{n-i}\left(\begin{array}{c}
n-i \\
j
\end{array}\right) s^{n-i-j} t^{j} V(K \underbrace{, \ldots,}_{n-i-j} K, L \underbrace{, \ldots,}_{j} L, E \underbrace{\ldots, \ldots, E}_{i}) .
\end{aligned}
$$

Throughout the discussion of parallel bodies we will assume that $E$ is a normal convex body in $E^{n}$. The relative inner parallel body of a convex body $K$ with respect to $E$ at distance $r(K ; E)-\lambda, 0 \leq \lambda \leq r(K ; E)$, is defined by

$$
K_{\lambda}=\{x: x+(r(K ; E)-\lambda) E \subseteq K\} .
$$

The inradius of $K_{\lambda}$ is $\lambda$. The kernel, $K_{0}$, is the inner parallel body of $K$ at the distance $r(K ; E)$, and $K=K_{r(K ; E)}$. The properties of inner parallel bodies have been studied by several authors, for example Bol [5], and Dinghas [9]. Many of their results may be found in [16, pp. 17-19, 60-70] and [17, pp. 147-150, 269-270, 274-276]. Properties of relative inner parallel bodies in the plane may also be found in $[8]$.

The following are immediate consequences of the definition of relative inner parallel bodies. For all $\lambda, 0 \leq \lambda \leq r(K ; E)$,

$$
K_{\lambda}+(r(K ; E)-\lambda) E \subseteq K
$$

and

$$
K_{\lambda}=\bigcap_{u \in U}\{x:\langle x, u\rangle \leq h(K, u)-(r(K ; E)-\lambda) h(E, u)\} .
$$

The family of inner parallel bodies is concave, see [16, p. 62] and [17, pp. 149-150], that is

$$
K_{\theta s+(1-\theta) t} \supseteq \theta K_{s}+(1-\theta) K_{t}, \quad 0 \leq \theta \leq 1,0 \leq s<t \leq r(K ; E) .
$$


Theorem (8) and (11) imply that the $(n-i)$ th root of the $i$ th relative quermassintegral is a concave function for $i=0, \ldots, n-2$. The $(n-1)$ st quermassintegral is concave and the $n$th is constant. Therefore the relative quermassintegrals are absolutely continuous functions, and their right- and left-hand derivatives exist everywhere on the half-open intervals $(0, r(K ; E)]$ and $[0, r(K ; E))$ respectively, and are equal almost everywhere. If $F^{\prime}(t)$ denotes the left-hand derivative of any function $F(t)$ with respect to $t$, then

$$
V^{\prime}\left(K_{t}\right)=n W_{1}\left(K_{t} ; E\right)
$$

and

$$
W_{i}^{\prime}\left(K_{t} ; E\right) \geq(n-i) W_{i+1}\left(K_{t} ; E\right), \quad i=1, \ldots, n-1 .
$$

The following lemma will also be needed.

(14) LemMA. Let $K$ and $E$ be convex bodies in $E^{n}, E$ normal. Then for $0 \leq s<t \leq r(K ; E)$,

$$
(t-s) W_{2}^{\prime}\left(K_{t} ; E\right) \leq(n-2)\left(W_{2}\left(K_{t} ; E\right)-V\left(K_{t}, \ldots, K_{t}, K_{s}, E, E\right)\right) .
$$

Equality holds if $K=K_{0}+r(K ; E) E$.

Proof. Let $r=r(K ; E)$, and choose $s$ and $t$ where $0 \leq s<t \leq r$. The definition of the left-hand derivative states

$$
W_{2}^{\prime}\left(K_{t} ; E\right)=\lim _{h \rightarrow 0}\left(W_{2}\left(K_{t} ; E\right)-W_{2}\left(K_{t-h} ; E\right)\right) / h .
$$

For $0 \leq h \leq t-s$, the concavity of the family of parallel bodies (11) implies

$$
K_{t-h} \supseteq \theta K_{s}+(1-\theta) K_{t}, \quad \theta=h /(t-s) .
$$

We will obtain a polynomial of degree $n-2$ in $h$ if we use (9) to expand the second quermassintegral of the convex combination in (16). To obtain the bound for the left-hand derivative of $W_{2}\left(K_{t} ; E\right)$ in (15) we need only determine the coefficients of $h$ to the zeroth and first powers. The first two terms of the polynomial are:

$$
W_{2}\left(K_{t} ; E\right)-h \frac{(n-2)}{t-s}\left(W_{2}\left(K_{t} ; E\right)-V\left(K_{t}, \ldots, K_{t}, K_{s}, E, E\right)\right) .
$$

This completes the proof of (15).

If $K=K_{0}+r E$ then (10) may be used to show that for all $t, 0 \leq t \leq r, K_{t}=$ $K_{0}+t E$. In this case equality holds in (16) and therefore equality holds in (15).

The conditions for equality in (13) remain unknown since they were first investigated by Bol, and the author does not know the necessary condition for equality in (15). For $n=3$, these conditions were established in [19] by considering the form body of the inner parallel bodies. Consideration of the form bodies may also result in sharper inequalities for bodies with "corners and edges". Examples of this type of result may be found in Bol [5], Bol and Knothe [6], Dinghas [9; 16, pp. 68-70; 17, pp. 274-276] and Theorem (19) below. The relative form body of $K$ about $E$ is defined by

$$
\bar{K}=\bigcap_{u \in U}\{x:\langle x, u\rangle \leq h(E, u)\} .
$$

It follows from the definition of the inner parallel body and the form body that

$$
K_{\lambda}+(r(K ; E)-\lambda) \bar{K} \subseteq K .
$$


Because all of the extreme supporting hyperplanes of the form body are also supporting hyperplanes of $E$, the form bodies are tangent bodies of $E$. Equivalent definitions of tangent bodies of various orders may be found in Favard [13, pp. 273-276] and Schneider [20]. In this discussion Schneider's numeration for tangent bodies will be used; the only property of tangent bodies needed is the following result of Favard.

(18) THEOREM (FAVARD). If $K$ and $E$ are two convex bodies in $E^{n}$, then

$$
V(K)=W_{i}(K ; E)
$$

if and only if $K$ is an $(n-i)$-tangent body of $E$.

IV. Relative Bonnesen inequalities.

(19) Theorem. Let $K$ and $E$ be convex bodies in $E^{n}$. Then

$$
\left(\frac{W_{1}(K ; E)}{W_{1}(\bar{K} ; E)}\right)^{n /(n-1)}-\frac{V(K)}{V(\bar{K})} \geq\left(\left(\frac{W_{1}(K ; E)}{W_{1}(\bar{K} ; E)}\right)^{1 /(n-1)}-r(K ; E)\right)^{n} .
$$

If $E$ is regular equality holds if and only if $K$ is a homothet of $\bar{K}$.

PROOF. It suffices to let $E$ be normal. Let $r=r(K ; E)$ and choose $\lambda, 0 \leq \lambda \leq r$. If we apply (17) to Theorem (8), for $i=1$, we have

$$
W_{1}\left(K_{\lambda} ; E\right) \leq\left(\sqrt[n-1]{W_{1}(K ; E)}-(r-\lambda) \sqrt[n-1]{W_{1}(\bar{K} ; E)}\right)^{n-1} .
$$

Integrating both sides from 0 to $r$ with respect to $\lambda$, from (12) and the fact that $V\left(K_{0}\right)=0$, we have

$$
\begin{aligned}
& \frac{1}{n} V(K) \leq \int_{0}^{r}\left(\sqrt[n-1]{W_{1}(K ; E)}-(r-\lambda) \sqrt[n-1]{W_{1}(\bar{K} ; E)}\right)^{n-1} d \lambda \\
&=\frac{1}{n \sqrt[n-1]{W_{1}(\bar{K} ; E)}(}\left(\sqrt[n-1]{W_{1}(K ; E)}\right)^{n} \\
&\left.-\left(\sqrt[n-1]{W_{1}(K ; e)}-r \sqrt[n-1]{W_{1}(\bar{K} ; E)}\right)^{n}\right) .
\end{aligned}
$$

Since $\bar{K}$ is an $(n-1)$-tangent body of $E$, Favard's Theorem (18) states $V(\bar{K})=$ $W_{1}(\bar{K} ; E)$. The inequality is established by dividing both sides by this quantity.

Assume $E$ is regular. Equality will hold if and only if equality holds in (20) for almost all $\lambda, 0 \leq \lambda \leq r(K ; E)$. If equality holds in (20) for some value of $\lambda$ on the open interval $(0, r(K ; E))$, then Aleksandrov's Theorem implies $K_{\lambda}$ and $\bar{K}$ are homothetic and equality holds in (17). This implies that $K$ and $\bar{K}$ are homothetic. On the other hand, if $K$ and $\bar{K}$ are homothetic then $K$ is a form body of some homothet of $E$. It suffices to consider the case where $K=\bar{K}$. If $u$ is an extreme direction of any form body $\bar{K}$ then $h(\bar{K}, u)=h(E, u)$. Therefore in this case, $h(K, u)=h(E, u)$ for all $u$ in $U$ and the representation of the inner parallel bodies in (10) implies that $K_{\lambda}=\lambda K$, for $0<\lambda \leq 1=r(K ; E)$. Therefore the inner parallel bodies are homothetic to $K$ and equality holds in (17). By Theorem (8) equality holds in (20) for all $\lambda$ in the half-open interval. 
A weaker version of this result may be obtained by replacing $\bar{K}$ with $E$ throughout the theorem and its proof. For $E=B$ the next corollary is the inequality of Hadwiger and Dinghas (3).

(21) COROLlaRY. Let $K$ and $E$ be convex bodies in $E^{n}$. Then

$$
\left(\frac{W_{1}(K ; E)}{V(E)}\right)^{n /(n-1)}-\frac{V(K)}{V(E)} \geq\left(\left(\frac{W_{1}(K ; E)}{V(E)}\right)^{1 /(n-1)}-r(K ; E)\right)^{n} \text {. }
$$

If $E$ is regular, equality holds if and only if $K$ is homothetic to $E$.

We may now use (1) to obtain a circumradius result from Corollary (21). Interchange $K$ and $E$ and use

$$
W_{i}(K ; E)=W_{n-i}(E ; K) .
$$

(22) COROLlary. Let $K$ and $E$ be convex bodies in $E^{n}$. Then

$$
\left(\frac{W_{n-1}(K ; E)}{V(K)}\right)^{n /(n-1)}-\frac{V(E)}{V(K)} \geq\left(\left(\frac{W_{n-1}(K ; E)}{V(K)}\right)^{1 /(n-1)}-\frac{1}{R(K ; E)}\right)^{n} \text {. }
$$

If $E$ is regular, equality holds if and only if $K$ is homothetic to $E$.

This completes the presentation of Bonnesen-style inequalities which sharpen the isoperimetric inequality. The next inequalities involve polynomials whose coefficients are relative quermassintegrals as in (4). A discussion of Theorem (23) and its corollaries will precede the proof.

(23) ThEOREM. Let $K$ and $E$ be convex bodies in $E^{n}, E$ normal. Then for all $\lambda, 0 \leq \lambda \leq r(K ; E)=r$

$$
\begin{aligned}
V\left(K_{\lambda}\right) \geq & V(K)-n(r-\lambda) W_{1}(K ; E)+(n-1)(r-\lambda)^{2} W_{2}(K ; E) \\
& +(n-1)(n-2) \int_{\lambda}^{r}(s-\lambda) V\left(K_{s}, \ldots, K_{s}, K_{\lambda}, E, E\right) d s .
\end{aligned}
$$

Equality holds for all $\lambda, 0 \leq \lambda \leq r$, if $K=K_{0}+r E$.

Evaluating (24) at $\lambda=0$ gives:

$$
\begin{aligned}
0 \geq & V(K)-n r W_{1}(K ; E)+(n-1) r^{2} W_{2}(K ; E) \\
& +(n-1)(n-2) \int_{0}^{r} s V\left(K_{s}, \ldots, K_{s}, K_{0}, E, E\right) d s .
\end{aligned}
$$

The definite integral in (25) is positive; therefore a stronger inequality than (4) is obtained as a corollary. The sufficient condition for equality follows from the Favard's Theorem (18).

(26) COROllary. Let $K$ and $E$ be convex bodies in $E^{n}$. Then

$$
0 \geq V(K)-n r W_{1}(K ; E)+(n-1) r^{2} W_{2}(K ; E) .
$$

Equality holds if $K$ is an $(n-2)$-tangent body of $E$.

By interchanging $K$ and $E$ and applying (1), as was done to obtain Corollary (22), a circumradius inequality is also obtained. By continuity this inequality is valid, and all terms are well defined, if $K$ is a bounded convex set with dimension less than or equal to $n$. 
(27) COROLlaRY. Let $K$ be a bounded convex set, $E$ a convex body in $E^{n}$ and $R(K ; E)=R$. Then

$$
0 \geq(n-1) W_{n-2}(K ; E)-n R W_{n-1}(K ; E)+R^{2} V(E) .
$$

Equality holds if $E$ is an $(n-2)$-tangent body of $K$.

If we consider the original Bonnesen inequality (2) it is obvious to conjecture that for $n>2$ the "alternating Steiner polynomial",

$$
\sum_{i=0}^{n}\left(\begin{array}{l}
n \\
i
\end{array}\right)(-t)^{i} W_{i}(K ; E),
$$

is negative on the closed interval $[r, R]$. This conjecture is false and counterexamples are given in a previous paper by the author [19]. In private communication, Bokowski observed that the Bonnesen-style inequalities, (3), (4) and (7), lead one to search for inequalities involving three quermassintegrals. To obtain (25) the author took the strongest linear inequality for three quermassintegrals, from Corollary (26), and added the term (in the form of a definite integral) which gave equality for bodies of the form $K_{0}+r E$. For $n=3$ the definite integral in (25) is equal to $r^{2} V\left(K_{0}, E, E\right)$. The author has not discovered an equally nice interpretation for this integral in higher dimensions.

Proof of THEOREM (23). Fix $\lambda, 0<\lambda<r$. For all $t, \lambda \leq t \leq r$ let

$$
\begin{aligned}
f(t)= & V\left(K_{\lambda}\right)-V\left(K_{t}\right)+n(t-\lambda) W_{1}\left(K_{t} ; E\right)-(n-1)(t-\lambda)^{2} W_{2}\left(K_{t} ; E\right) \\
& -(n-1)(n-2) \int_{\lambda}^{t}(s-\lambda) V\left(K_{s}, \ldots, K_{s}, K_{\lambda}, E, E\right) d s .
\end{aligned}
$$

Using (12) we find

$$
\begin{aligned}
f^{\prime}(t)= & n(t-\lambda) W_{1}^{\prime}\left(K_{t} ; E\right)-2(n-1)(t-\lambda) W_{2}\left(K_{t} ; E\right) \\
& -(n-1)(t-\lambda)^{2} W_{2}^{\prime}\left(K_{t} ; E\right) \\
& -(n-1)(n-2)(t-\lambda) V\left(K_{t}, \ldots, K_{t}, K_{\lambda}, E, E\right) \\
= & n(t-\lambda)\left(W_{1}^{\prime}\left(K_{t} ; E\right)-(n-1) W_{2}\left(K_{t} ; E\right)\right) \\
& +(n-1)(t-\lambda)\left((n-2)\left(W_{2}\left(K_{t} ; E\right)-V\left(K_{t}, \ldots, K_{t}, K_{\lambda}, E, E\right)\right)\right. \\
& \left.\quad-(t-\lambda) W_{2}^{\prime}\left(K_{t} ; E\right)\right)
\end{aligned}
$$

Therefore by (13) and Lemma (14) $f^{\prime}(t) \geq 0 ; f(\lambda)=0$, implies $f(r) \geq 0$. This establishes the inequality.

If $K=K_{0}+r E$, then $K_{\lambda}=K_{0}+\lambda E$ for $0 \leq \lambda \leq r$. The volume of $K_{\lambda}$ and the mixed volume of $K_{s}, K_{\lambda}$ and $E$ may be expanded using (9). Equality follows.

V. Conclusion. First, bounds for the inradius and circumradius will be discussed. The inequalities in the four corollaries yield bounds for $r(K ; E)$ and $R(K ; E)$; the corollaries to Theorem (19) give only a lower bound for the inradius and an upper bound for the circumradius. Additional bounds are

$$
\begin{gathered}
V(K) / W_{1}(K ; E) \geq r(K ; E), \\
R(K ; E) \geq W_{n-1}(K ; E) / V(E),
\end{gathered}
$$

and those which may be obtained from $(7)$ for $R(K ; B)$. It is a simple matter to show that the quotient of volume and the first relative quermassintegral is a better 
upper bound for the inradius than the upper bound obtained from Corollary (26); this is analogous to the upper bound in (5). Equality holds if and only if $K$ is an $(n-1)$-tangent body of $E$. The corollaries give two lower bounds for $r$ and two upper bounds for $R$; in general neither is better than the other. For example if $E=B$, the lower bound for the inradius in Corollary (21) is better for a ball-like body, while the lower bound in Corollary (26) is better for pencil shaped bodies. It remains to consider lower bounds for $R(K ; E)$. In relative geometry the quotient of the $(n-1)$ st relative quermassintegral and the volume of $E$ is best. If $E=B$, then a better bound is obtained from (7) analogous to (6), see $[\mathbf{4}, \mathrm{p} .7]$.

Next, the following conjecture is due to Teissier $\left[\mathbf{2 1}, \mathrm{p}\right.$. 101]. Let $s_{1} \leq \cdots \leq s_{n}$ be the real parts of the roots of the alternating Steiner polynomial (2.8). Then

$$
0<s_{1} \leq r(K ; E), \quad \text { and } R(K ; E) \leq s_{n} .
$$

Notice that this conjecture would provide a third lower bound for $r$, a third upper bound for $R$, and if $n=2$ it reduces to (2). It is simple to show, for $n=3$, that all roots of (28) lie in the positive real half-plane, and if $E=B$ and $K$ is a cap body (1-tangent body of $B$ ) then (28) does have complex roots.

Finally, let $\Phi_{i}(K ; E)=R^{i}(K ; E) W_{i}(K ; E)$ and $\varphi_{i}(K ; E)=r^{i}(K ; E) W_{i}(K ; E)$. The inequalities of Bokowski and Heil (7) are upper bounds for $\Phi_{j}(K ; B)$ by a weighted arithmetic mean of $\Phi_{i}(K ; B)$ and $\Phi_{k}(K ; B), 0 \leq i<j<k \leq n$. Notice that Corollaries (26) and (27) are similar inequalities, but lower bounds for $\varphi_{1}(K ; E)$ and $\Phi_{n-1}(K ; E)$. Bokowski and Heil show, by considering truncated spheres, that the weights which appear in their inequalities are best. One may show for a family of convex bodies, say cylinders, which converge to an $(n-1)$ dimensional ball that Corollary (26) for $E=B$ is best. Unlike (7), Corollaries (26) and (27) are not special cases of a larger family of inequalities involving any three relative quermassintegrals. To see this consider the circumradius. The only valid inequalities are

$$
0 \geq(n-1) \Phi_{i}(K ; E)-n \Phi_{n-1}(K ; E)+\Phi_{n}(K ; E),
$$

for $i=0, \ldots, n-2$, which follow directly from Corollary (27). Suppose there were another inequality where $\Phi_{j}(K ; E)$ is bounded below by a weighted arithmetic mean of $\Phi_{i}(K ; E)$ and $\Phi_{k}(K ; E)$ for $0 \leq i<j<k \leq n, n>2$ and $j<n-1$. Let $K$ be an $(n-1-j)$-dimensional convex set. Then its $i$ th and $j$ th quermassintegrals are zero, its $k$ th quermassintegral is positive and since $K$ has dimension at least 1 , its circumradius is positive. Because of the direction of the inequality sign, the inequality is invalid.

\section{REFERENCES}

1. A. D. Aleksandrov, On the theory of mixed volumes (Russian), translated by W. J. Firey, Oregon State Univ., Corvallis.

2. W. Blaschke, Vorlesungen über Integralgeometrie. I, II, Hamb. Math. Einzel. 20, 22, Teubner, Leipzig, 1936-1937; reprint, Chelsea, New York, 1949.

3. J. Bokowski, Eine verschärfte Ungleichung zwischen Volumen, Oberfläche und Inkugelradius im $R^{n}$, Elem. Math. 28 (1973), 43-44.

4. J. Bokowski and E. Heil, Integral representations of quermassintegrals and Bonnesen-style inequalities, Arch. Math. (to appear)

5. G. Bol, Beweis einer Vermutung von H. Minkowski, Abh. Math. Sem. Univ. Hamburg 15 (1943), 37-56. 
6. G. Bol and H. Knothe, Über konvexe Körper mit Ecken und Kanten, Arch. Math. 1 (19481949), 427-431.

7. T. Bonnesen, Les problèmes des isopérimètres et des isèpiphanes, Gauthier-Villars, Paris, 1929.

8. G. D. Chakerian and J. R. Sangwine-Yager, A generalization of Minkowski's inequality for plane convex sets, Geom. Dedicata 8 (1979), 437-444.

9. A. Dinghas, Bemerkung zu einer Verschärfung der isoperimetrischen Ungleichung durch H. Hadwiger, Math. Nachr. 1 (1948), 284-286.

10. V. I. Diskant, A generalization of Bonnesen's inequalities, Soviet Math. Dokl. 14 (1973), no. 6, 1728-1731 (transl. of Dokl. Akad. Nauk SSSR 213 (1973), no. 3).

11. H. G. Eggleston, Convexity, Cambridge Univ. Press, Cambridge, 1969.

12. J. Favard, Problèmes d'extremums relatifs aux courbes convexes. I, Ann. Sci. École Norm. Sup. 46 (1929), 345-369.

13. __ Sur les corps convexes, J. Math. Pures Appl. (9) 12 (1933), 219-282.

14. H. Flanders, A proof of Minkowski's inequality for convex curves, Amer. Math. Monthly 75 (1968), 581-593.

15. H. Hadwiger, Die isoperimetrische Ungleichung im Raum, Elem. Math. 3 (1948), 25-38.

16. __ Altes und Neues über konvexe Körper, Birkhäuser Verlag, Basel, 1955.

17. __ Vorlesungen über Inhalt, Oberfläche und Isoperimetrie, Springer, Berlin, 1957.

18. R. Osserman, Bonnesen-style isoperimetric inequalities, Amer. Math. Monthly 86 (1979), 1-29.

19. J. R. Sangwine-Yager, A Bonnesen-style inradius inequality in 3-space, Pacific J. Math. (to appear).

20. R. Schneider, Über Tangentialkörper der Kugel, Manuscripta Math. 23 (1978), 269-278.

21. B. Teissier, Bonnesen-type inequalities in algebraic geometry. I: Introduction to the problem, Seminar on Differential Geometry, Princeton Univ. Press, 1982, pp. 85-105.

22. J. M. Willis, Zum Verhältnis von Volumen zur Oberfläche bei konvexen Körpern, Arch. Math. 21 (1970), 557-560.

Department of Mathematics, Saint Mary's College of California, Moraga, CALIFORNIA 94575 